\title{
Myocardial T1-mapping for early detection of left ventricular myocardial fibrosis in systemic sclerosis
}

\author{
Franck Thuny ${ }^{1 *}$, Leila Potton ${ }^{2}$, Stanislas Rapacchi ${ }^{3}$, Hélène Thibault ${ }^{4}$, Cyrille Bergerot ${ }^{4}$, Magalie Viallon ${ }^{5}$, \\ Nathan Mewton ${ }^{4}$, Michel Ovize ${ }^{4}$, Geneviève Derumeaux ${ }^{4}$, Pierre Croisille ${ }^{1}$
}

From 2011 SCMR/Euro CMR Joint Scientific Sessions

Nice, France. 3-6 February 2011

\section{Background}

Subclinical primary left ventricular (LV) dysfunction is a complication of systemic sclerosis (SSc) related to progressive diffuse myocardial fibrosis. Myocardial T1-mapping has recently been proposed as a cardiac magnetic resonance (CMR) method to quantify interstitial fibrosis early in the disease course whereas late-gadolinium enhancement (LGE) imaging remains normal.

\section{Objective}

We aimed to evaluate whether myocardial T1-mapping could detect subclinical abnormalities in SSc patients with normal standard parameters of LV function and normal LGE imaging.

\section{Methods}

We prospectively studied 37 patients ( $52 \pm 12$ years) presenting with SSc with no history of heart disease, no pulmonary hypertension at rest, a normal LV assessed by conventional echocardiography (normal volumes, ejection fraction and wall motion), and no LGE. SSc patients were compared to16 matched controls healthy volunteers ( $47 \pm 7$ years old). T1 quantification was performed using a Modified Look-Locker Inversionrecovery (MOLLI) sequence at $1.5 \mathrm{~T}$ (Siemens) on a LV short axis before, $5 \mathrm{~min}$ and $15 \mathrm{~min}$ after $0.2 \mathrm{mmol} / \mathrm{Kg}$ gadolinium injection. Imaging protocol included also standard Cine-SSFP imaging, and LGE imaging. LV diastolic function (mitral inflow pattern) was further assessed using echocardiography.

\section{Results}

A non significant shorter mean T1 time (ms, mean \pm SEM) was observed in SSc patients compared to controls both at $5(357 \pm 5$ vs. $361 \pm 6, \mathrm{p}=0.65)$ and $15(448 \pm 5$ vs. $456 \pm 5, \mathrm{p}=0.34)$ minutes after gadolinium injection. Echocardiography displayed a LV diastolic dysfunction in $47 \%$ of SSc patients and in $25 \%$ of controls $(\mathrm{p}=0.04)$. The SSc patients with a LV diastolic dysfunction had a shorter 15 minutes post-contrast $\mathrm{T} 1$ time $(\mathrm{ms})$ than those with a normal diastolic function $(431 \pm 7$ vs. $464 \pm 8$, $\mathrm{p}=0.01$ ).

\section{Conclusions}

Post-contrast T1-mapping identifies changes in LV myocardial T1 times in patients with SSc, normal LVEF and impaired LV diastolic function. These abnormalities may reflect the impact of diffuse interstitial myocardial fibrosis in SSc that could be early detected in the future.

\section{Author details}

${ }^{1}$ Hopital Louis Pradel-Laboratoire Creatis-LRMN-UMR CNRS 5220 \& INSERM U630, Lyon, France. ${ }^{2} \mathrm{CHU}$ de Grenoble, Grenoble, France. ${ }^{3}$ Laboratoire Creatis-LRMN-UMR CNRS 5220 \& INSERM U630, Lyon, France. ${ }^{4}$ Hopital Louis Pradel, Lyon, France. ${ }^{5}$ University Hospitals of Geneva, Geneve, Switzerland.

Published: 2 February 2011

doi:10.1186/1532-429X-13-S1-M10

Cite this article as: Thuny et al: Myocardial T1-mapping for early detection of left ventricular myocardial fibrosis in systemic sclerosis. Journal of Cardiovascular Magnetic Resonance 2011 13(Suppl 1):M10. 